Artigo / Article

\title{
Tratamento de primeira linha da leucemia linfóide crônica
}

Chronic lymphocytic leukemia: first line treatment

Carlos S. Chiattone

\begin{abstract}
A leucemia linfóide crônica (LLC-B) é uma neoplasia de comportamento indolente, que o tratamento imediato não mostrou vantagem em prolongar a sobrevida dos pacientes em estádios iniciais. Entretanto, diversos agentes são efetivos no tratamento desta doença. Agentes alquilantes, análogos de purina e anticorpos monoclonais são os mais utilizados, determinando altas taxas de resposta em pacientes tratados ou não previamente. Baseado em efeito sinérgico observado in vitro, diversos estudos têm usado estes agentes de forma combinada. Análogos de purina usados de forma isolada ou combinada com agentes alquilantes e anticorpos monoclonais determinam taxas de resposta superiores comparadas com as de agentes alquilantes isolados. Entretanto, apesar do expressivo aumento nas taxas de resposta, estas novas modalidades de tratamento não prolongam a sobrevida e a $L L C$-B permanece incurável. Rev. bras. hematol. hemoter. 2005;27(4):276-279.
\end{abstract}

Palavras-chave: Leucemia linfóide crônica; tratamento.

Apesar do recente avanço no conhecimento dos aspectos biológicos, a leucemia linfóide crônica (LLC-B) continua sendo uma doença incurável com o tratamento atualmente disponível. ${ }^{1} \mathrm{O}$ tratamento convencional freqüentemente acarreta remissão da doença, embora ocorra recidiva virtualmente em todos os casos. $\mathrm{O}$ uso indiscriminado de quimioterapia para todos os pacientes em estádio inicial da LLC-B induz apenas toxicidade, sem vantagem na sobrevida. $\mathrm{O}$ tratamento para estes pacientes não está indicado, exceto em contexto de estudo clínico., ${ }^{2,3}$

Como discutido em "indicações para início do tratamento", o procedimento de observação (watchful waiting) permanece a conduta convencional para os pacientes assintomáticos em estádio inicial. ${ }^{2-5}$ Uma metanálise de seis estudos randomizados, envolvendo 2.048 pacientes em estádios iniciais (Rai menor ou igual a II; Binet A) indicou que tratamento imediato com clorambucil, associado ou não a prednisona, não era superior à conduta de iniciar o tratamento apenas no momento da progressão da doença (sobrevida aos 10 anos: $44 \%$ versus $47 \%$, respectivamente, $p>0,1){ }^{5}$
Se não houver necessidade de tratamento imediato, os pacientes podem ser reavaliados a cada três a seis meses (pacientes bem orientados quanto à identificação de manifestações sugestivas de progressão da doença podem ser acompanhados com intervalos maiores). O paciente é monitorado para o aparecimento de organomegalia ou citopenia e pelo cálculo do tempo de duplicação do número de linfócitos. Para pacientes com características de alto risco (ex.: deleção de $17 p$ ou 11q) é recomendado intervalo mais curto entre as consultas. É importante destacar que o número de linfócitos, isoladamente, não é indicação de início de tratamento, pois, diferente das leucemias agudas e da leucemia mielóide crônica, é extremamente rara a ocorrência de hiperviscosidade ou leucoestase em decorrência da hipercelularidade na LLC-B. ${ }^{6}$

Diversos estudos têm investigado o tratamento precoce para pacientes assintomáticos em estádio inicial, mas apresentando fatores prognósticos desfavoráveis, como expressão de $\mathrm{CD} 38$, estado não mutado de genes $\operatorname{IgVH}$ ou alterações citogenéticas negativas. Como estes casos de mau

Disciplina de Hematologia e Oncologia,FCM da Santa Casa de São Paulo.

Hemocentro da Santa Casa de São Paulo.

Correspondência para: Carlos S. Chiattone

Faculdade de Ciências Médicas da Santa Casa de São Paulo, Disciplina de Hematologia e Oncologia. Hemocentro da Santa Casa de São Paulo.

Rua Marquês de Itu, 579

01223-001 - São Paulo - SP, Brasil

E-mails:dir.bs@santacasasp.org.br; carlos.chiattone@terra.com.br 
prognóstico podem apresentar sobrevida muito curta, mesmo quando são diagnosticados em estádio clínico inicial, é aconselhável encaminhar estes pacientes para estudo clínico, quando disponível.

Atualmente, há uma variedade de opções terapêuticas para os pacientes com LLC-B que requerem tratamento. ${ }^{7}$ Estas incluem: agentes alquilantes orais (como clorambucil ou ciclofosfamida), análogos de purina (fludarabina, cladribina e pentostatina), poliquimioterapia (como CVP ou CHOP), anticorpos monoclonais, quimioimunoterapia e mesmo transplante de células-tronco hematopoéticas.

O tratamento com clorambucil induz taxa de resposta de $25 \%$ a $35 \%$; entretanto, resposta completa é observada em somente cerca de $3 \%$, com curta duração da remissão (mediana de cerca de 14 meses) ${ }^{8,9} \mathrm{O}$ tratamento é bem tolerado, com moderada mielotoxicidade. Associação de clorambucil com prednisona não é vantajosa em relação ao uso isolado do clorambucil, exceto quando a LLC-B é acompanhada de manifestação auto-imune. ${ }^{10}$ Em decorrência de sua fácil utilização, baixo custo e relativamente baixa taxa de complicação, o clorambucil ainda pode ser considerado a primeira opção para pacientes com idade muito avançada, especialmente naqueles com comorbidades. ${ }^{11}$ Em pacientes mais jovens, com sintomas que demandam indução de remissão mais efetiva, a tendência é para o uso de fludarabina. ${ }^{7}$

Os seguintes esquemas para a utilização de clorambucil têm sido descritos:

- Intermitente: $0,4 \mathrm{mg} / \mathrm{kg}$ no dia 1 , cada 14 dias, com possível aumento da dose em $0,1 \mathrm{mg} / \mathrm{kg}$ em cada ciclo se não houver remissão (dose máxima: $0,8 \mathrm{mg} / \mathrm{kg})^{12}$

- Intermitente: $40 \mathrm{mg} / \mathrm{m}^{2}$ no dia 1 , cada 28 dias $^{8}$

- Intermitente mais prednisona: $0,3 \mathrm{mg} / \mathrm{kg}$ nos dias 15 , cada 28 dias, e prednisona $40 \mathrm{mg} / \mathrm{m}^{2}$ nos dias $1-5$, cada 28 dias $^{3}$

- Contínuo, baixa dose: $0,1 \mathrm{mg} / \mathrm{kg}$ diariamente ${ }^{3}$

- Contínuo, alta dose: $15 \mathrm{mg} /$ dia até toxicidade (obs: alta toxicidade freqüente), máximo de 6 meses $^{13}$

O tratamento com clorambucil deve ser mantido até que o efeito máximo seja obtido. É importante ressaltar que terapia de consolidação com esta droga não determina benefício ao paciente. Se o intervalo livre de progressão após o uso de clorambucil for maior que 6 a 12 meses, a mesma droga poderá ser usada no retratamento. Se a progressão ocorre durante, ou logo após o uso do clorambucil, o tratamento deve ser mudado para outra droga quimioterápica, como a fludarabina.

O tratamento com poliquimioterapia não é vantajoso em relação ao uso do clorambucil isolado. Estudos comparando CVP (ciclofosfamida, vincristina e prednisona) com clorambucil mostram taxas de resposta semelhantes. ${ }^{14,15}$ Quando a quimioterapia contém antraciclina, a taxa de resposta é geralmente maior, mas com aumento dos efeitos colaterais e sem vantagem na sobrevida. ${ }^{16,17}$
Nos últimos anos, especialmente na última década, o tratamento da LLC-B sofreu um avanço com o uso de análogos de purina (fludarabina, cladribina e pentostatina), usados de forma isolada ou combinada com outros agentes. Entre os análogos de purina, a fludarabina é a droga que tem sido usada mais extensivamente. Fludarabina isolada, em pacientes previamente não tratados, determina taxa de resposta de $60 \%$ a $70 \%$, com resposta completa entre $20 \%$ e $30 \%{ }^{8}$

Em três estudos fase III de tratamento inicial de LLC$\mathrm{B}$, a fludarabina mostrou maior taxa de resposta global e resposta completa que qualquer outro esquema quimioterápico convencional como clorambucil, CAP (ciclofosfamida, adriblastina e prednisona) ou CHOP (ciclofosfamida, adriblastina, vincristina e prednisona). ${ }^{8,18,19}$ Comparado com estes tratamentos, a fludarabina mostra uma evidente vantagem quanto à sobrevida livre de progressão; entretanto, embora haja uma tendência, não se observa vantagem estatisticamente significante na sobrevida global, possivelmente em decorrência da permissão, nestes estudos, de crossover na recaída. ${ }^{6}$

Em decorrência da possibilidade de agravamento de hemólise auto-imune com uso de fludarabina, é altamente recomendável a realização de teste de Coombs direto antes do tratamento. Se o teste for positivo ou houver histórico ou presença de anemia hemolítica auto-imune (AHAI), embora controverso, não é aconselhável o seu uso. Nestes casos, devese procurar a negativação do teste de Coombs com uso de corticosteróide antes da introdução da fludarabina. Uma metanálise, incluindo cinco estudos envolvendo aproximadamente 1.300 pacientes, indicou que a fludarabina, comparada com diversos regimes quimioterápicos baseados em agentes alquilantes, determina maior taxa de infecção, embora com igual taxa de morte em decorrência desta complicação. ${ }^{20}$

Com outros análogos de purina, além da fludarabina, há menos experiência relatada na literatura. Estudo fase III comparando cladribina com clorambucil, associados a prednisona, mostrou maior taxa de resposta completa $(47 \%$ versus $12 \%$, respectivamente). Não houve diferença, entretanto, na sobrevida global aos dois anos de seguimento. ${ }^{21}$

A fludarabina tem sido testada em diversas combinações. A mais freqüente destas é com a ciclofosfamida. ${ }^{22-25}$ Em estudos iniciais, a taxa de resposta não se mostrou diferente quando se usou fludarabina (F) isolada ou associada com a ciclofosfamida (C). No entanto, a qualidade da resposta foi melhor com o regime de associação das duas drogas. Esta combinação, associada ou não a mitoxantrone, mostra taxa de resposta de $64 \%$ a $100 \%$, com resposta completa de até $50 \% .^{22}$

A combinação FC associada a mitoxantrone foi testada pelo grupo de Barcelona em 37 pacientes com LLC-B refratária ou recidivada determinando alta taxa de resposta completa (50\%), incluindo dez casos com negativação de doença residual mínima, com duração da resposta de 19 
meses. ${ }^{26}$ A mediana de duração da resposta ainda não foi atingida pelos pacientes que obtiveram resposta completa, comparada com 25 meses dos pacientes nos quais o tratamento não determinou resposta completa. A combinação de cladribina com ciclofosfamida também se mostra muito ativa na LLC-B, mas estudo fase II mostra resultados inferiores à combinação fludarabina com ciclofosfamida (FC). ${ }^{27}$

O grupo alemão de estudo de LLC-B (GCLLSG) comparou, prospectivamente, fludarabina com a associação FC, em 375 pacientes virgens de tratamento. $\mathrm{O}$ estudo mostrou taxa de resposta superior no braço da associação $\mathrm{FC} .{ }^{28} \mathrm{~A}$ combinação FC demonstrou maior taxa de resposta completa $(16 \%)$ e resposta global $(94 \%)$, comparada com o braço da fludarabina $(5 \%$ e $83 \%, p=0,004$ e 0,001 , respectivamente). $\mathrm{O}$ braço FC também evidenciou superioridade no tempo de duração da resposta (48 meses versus 20 meses; $\mathrm{p}=0,001)$ e na sobrevida livre de evento (49 meses versus 33 meses; $\mathrm{p}=0,001)$. Até o momento da última análise deste estudo (mediana de seguimento de 22 meses) não se observou diferença na sobrevida global. Este estudo evidenciou mais freqüentemente trombocitopenia e neutropenia no braço $\mathrm{FC}$, mas menos anemia. Os autores relatam que a combinação FC não ocasionou maior número de infecções graves.

Como os estudos iniciais em linfomas indolentes mostraram menor efetividade do rituximabe em linfomas de pequenos linfócitos (a contrapartida tumoral da LLC-B) que no linfoma folicular, apenas recentemente este anticorpo monoclonal voltou a despertar interesse nos investigadores de LLC-B. Este interesse é determinado principalmente pelos dados in vitro indicando importante sinergismo entre rituximabe e fludarabina. ${ }^{29}$

A maioria dos estudos utilizando rituximabe tem associado este anticorpo monoclonal com fludarabina ou com a combinação FC. Estudo alemão fase II do GCLLSG utilizou fludarabina com rituximabe em 31 pacientes com LLC$\mathrm{B}$, como primeira linha ou em recaída. ${ }^{30} \mathrm{~A}$ taxa de resposta foi de $87 \%$, sendo que $32 \%$ obtiveram resposta completa.

O grupo $\mathrm{CALGB}^{31}$ usou a fludarabina associada ao rituximabe, tanto em esquema concomitante como seqüencial, em estudo randomizado. Pacientes com LLC-B sem tratamento anterior $(\mathrm{N}=104)$ receberam seis ciclos de fludarabina, com ou sem rituximabe, seguido de uma infusão de rituximabe semanal, por quatro vezes (CALGB 9712). As taxas de resposta global e completa foram superiores no braço que recebeu rituximabe concomitante com a fludarabina ( $90 \%$ e $47 \%$ versus $77 \%$ e $28 \%$ ). Em análise retrospectiva, os pacientes deste estudo CALGB 9712, tratados com fludarabina e rituximabe, foram comparados com 178 pacientes de estudo anterior (CALGB 9011) que receberam apenas fludarabina. ${ }^{32}$ Os pacientes que receberam fludarabina com rituximabe tiveram melhor sobrevida livre de progressão (SLP) e sobrevida global (SG) que os pacientes que receberam apenas fludarabina. As taxas, aos dois anos, de SLP foi de $67 \%$ versus $45 \%$ e de SG de $93 \%$ versus $81 \%$.
Em outro estudo fase II, do MDACC, envolvendo 224 pacientes com LLC-B sem tratamento anterior, a associação de rituximabe com $\mathrm{FC}$ resultou em taxa de resposta de $95 \%$, com $71 \%$ de resposta completa. ${ }^{33}$ A mediana de sobrevida global ainda não foi atingida para os pacientes tratados com esta combinação, mas é significativamente mais longa quando comparada com dados históricos de pacientes tratados apenas com a combinação FC.

\section{Conclusões do II Encontro Brasileiro de Consenso em LLC-B}

1. Tratamento da doença inicial (Binet A ou B, sem sintomas; Rai 0,I ou II, sem sintomas)

- O procedimento convencional para doença inicial é apenas a estratégia de observação (watch \& wait) com controle do sangue periférico e exame clínico a cada três meses.

- O tratamento para os estádios iniciais é indicado apenas quando houver presença de sintomas relacionados à doença ou sinais de rápida progressão da LLC-B (como "tempo de duplicação de linfócitos" menor que 12 meses)

\section{Tratamento da doença avançada (Binet A ou B com} sintomas, Binet C; Rai 0-II com sintomas, Rai III-IV)

- Pacientes jovens ( $\leq 65$ anos, fisicamente ativo, sem comorbidade maior)

- Análogo de purina pode ser recomendado como tratamento inicial, pois determina maior taxa de resposta completa e mais longas sobrevida livre de progressão e sobrevida livre de tratamento que o clorambucil.

- Os riscos e benefícios da fludarabina estão mais bem documentados na literatura que os de outros análogos de purina.

- Combinações de análogo de purina com outras drogas, em particular com a ciclofosfamida, resultam em maior taxa de resposta completa que a terapia com análogo de purina isolado, mas com maior toxicidade.

- Uso de rituximabe associado a fludarabina e ciclofosfamida parece promissor

- Pacientes idosos ( > 65 anos, com comorbidade maior)

- Clorambucil deve ser utilizado como terapia de primeira linha, pois é menos mielotóxico e imunossupressor que os análogos de purina.

\footnotetext{
Abstract

Chronic lymphocytic leukemia (CLL-B) is a neoplasia of indolent behavior for which immediate treatment has not shown an advantage in prolonging the survival of patients in initial stages. However, various agents are effective in the treatment of this disease. Alkylating agents, purine analogs and monoclonal antibodies are the most widely used, giving high response rates in patients who have or have not been previously treated. Based on the synergetic effect observed in vitro, diverse studies have utilized
} 
these agents in a combined form. Purine analogs used in isolation or in combination with alkylating agents and monoclonal antibodies obtain better response rates in comparison to those of isolated alkylating agents. Nonetheless, in spite of the expressive increase in the response rates, these new modalities of treatment do not prolong survival and CLL-B remains incurable. Rev. bras. hematol. hemoter. 2005;27(4):276-279.

Key Words: Chronic lymphocytic leukemia; treatment.

\section{Referências Bibliográficas}

1. Dighiero G. CLL Biology and Prognosis. Hematology: 2005;278-284.

2. Shustik C, Mick R, Silver R et al. Treatment of early chronic lymphocytic leukemia: intermittent chlorambucil versus observation. Hematol Oncol 1988;6(1):7-12.

3. Dighiero G, Maloum K, Desablens B et al Chlorambucil in indolent chronic lymphocytic leukemia. French Cooperative Group on Chronic Lymphocytic Leukemia . N Engl J Med 1998;338(21):1.506-14.

4. Effects of chlorambucil and therapeutic decision in initial forms of chronic lymphocytic leukemia (stage A): results of a randomized clinical trial on 612 patients. The French Cooperative Group on Chronic Lymphocytic Leukemia. Blood 1990;75(5):1.414-21.

5. Chemotherapeutic options in chronic lymphocytic leukemia: a metaanalysis of the randomized trials. CLL Trialists' Collaborative Group. J Natl Cancer Inst 1999; 91(10):861-8

6. Shanafelt TD, Call TG. Current approach to diagnosis and management of chronic lymphocytic leukemia. Mayo Clin Proc 2004;79(3):388-98.

7. Hallek M. Chronic Lymphocytic Leukemia: first-line treatment. Hematology 2005;285-91.

8. Rai KR, Peterson BL, Appelbaum FR et al. Fludarabine compared with chlorambucil as primary therapy for chronic lymphocytic leukemia. $\mathrm{N}$ Engl J Med 2000;343(24): 1.750-7.

9. Schulz H, Klein SK, Rehwald U et al; German CLL Study Group. Phase 2 study of a combined immunochemotherapy using rituximab and fludarabine in patients with chronic lymphocytic leukemia. Blood 2002;100(9): 3.115-20.

10. Han T, Ezdinli EZ, Shiamaoka K et al. Chlorambucil versus combined chlorambucil-corticosteroid therapy in chronic lymphocytic leukemia. Cancer 1973;31(3): 502-8.

11. Schriever F, Huhn D. New directions in the diagnosis and treatment of chronic lymphocytic leukemia. Drugs 2003;63(10):953-69.

12. Knospe WH, Loeb Jr V, Hululey Jr CM. Bi-weekly chlorambucil treatment of chronic lymphocytic leukemia. Cancer 1974;33(2): 555-562.

13. Jaksic B, Brugiatelli M, Krc I et al. High dose chlorambucil versus Binet's modified cyclophosphamide, doxorubicin, vincristin, and prednisone regimen in the treatment of patients with advenced B-cell chronic lymphocytic leukemia: results of an international multicenter radomized trial. Cancer 1997;79(11):2.107-14.

14. A randomized clinical trial of chlorambucil versus $\mathrm{COP}$ in stage $\mathrm{B}$ chronic lymphocytic leukemia. The French Cooperative Group on Chronic Lymphocytic Leukemia. Blood 1990;75(7):1.422-5.

15. Liepman M, Votam ML. The treatment of chronic lymphocytic leukemia with COP chemotherapy. Cancer 1978;41(5):1.664-9.

16. Keating MJ, Scouros M, Murphy S et al. Multiple agent chemotherapy $(\mathrm{POACH})$ in previously treated and untreated patients with chronic lymphocytic leukemia. Leukemia 1988;2:157.

17. Kimby E, Mellstedt H. Chlorambucil/prednisone versus CHOP in syntomatic chronic lymphocytic leukemia of B-cell type. A randomized trial. Leuk Lymphoma 1991;5:93

18. Johnson S, Smith AG, Löffler H et al. Multicentre prospective randomized trial of fludarabina versus cyclophosphamide, doxorubicin, and prednisone
(CAP) for treatment of advanced-stage chronic lymphocytic leukemia. Lancet 1996;347(9013):1.432-8.

19. Leporrier M, Chevret S, Cazin Boudjerra B et al. Randomized comparation of fludarabine, $\mathrm{CAP}$, and $\mathrm{CHOP}$ in 938 previously untreated stage $\mathrm{B}$ and C chronic lymphocytic leukemia patients. Blood 2001;98(8): 2.319-25.

20. Zhu Q, Tan DCL, Samuel M, et al. Fludarabine in comparison to alkylatorbased regimen as induction therapy for chronic lymphocytic leucemia: A systematic review and meta-analysis. Leuk Lymphoma 2004;45:2.239-45.

21. Robak T, Blonski JZ, Kasznicki M. Cladribine with prednisone versus chlrambucil with prednisone as first-line therapy in chronic lymphocytic leukemia: report of a prospective, randomized, multicenter trial. Blood 2000;96:2.723-2729.

22. Hallek M, Eichhorst BF. Chemotherapy combination treatment regimens with fludarabina in chronic lymphocytic leukemia. Hematol J 2004;5 (suppl.1):S20-S30.

23. O'Brien SM, Kantarjian HM, Cortes J et al. Results of the fludarabine and cyclophosfphamide combination regimen in chronic lymphocytic leukemia. J Clin Oncol 2001;19(5):1.414-20.

24. Hallek M, Schmitt B, Wilhelm M et al. German Chronic. Lymphocytic Leukaemia Study Group. Fludarabine plus cyclophosphamide is an efficient treatment for advanced chronic lymphocytic leukaemia (CLL): results of a phase II study of the German CLL Study Group. BR J Haematol 2001;114(2):342-8.

25. Flinn IW, Byrd JC, Morrison C et al. Fludarabine and cyclophosphamide with filgastrim support in patients with previously untreated indolent lymphoid malignancies. Blood 2000;96(1):71-5.

26. Bosch F, Ferrer A, Lopez-Guillermo A et al. Fludarabine, cyclophosphamide and mitoxantrone in the treatment of resistant or relapsed chronic lymphocytic leukemia. Br J Haematol 2002;119:976-984.

27. Monttillo M, Tedeschi A, O'Brian S et al. Phase II study of cladribine and cyclophosphamide in patients with chronic lymphocytic leukemia and prolymphocytic leukemia. Cancer 2003;97:114-120.

28. Eichhorst B, Busch R, Hopfinger G, the GCLLSG. Fludarabine plus cyclophosphamide versus fludarabine alone in first line therapy of younger patients with chronic lymphocytic leukemia. Blood 2005: in press.

29. di Gaetano N, Xiao Y, Erba E. Synergism between fludarabine and rituximab revealed in a follicular lymphoma cell line resistant to the cytotoxic activity of either drug alone. Br J Haematol 2001;114:800-809.

30. Schulz H, Klein SH, Rehwald U et al. Phase II study of a combined immunochemotherapy using rituximab and fludarabine in patients with chronic lymphocytic leukemia. Blood 2002;100:3.115-3.120.

31. Byrd JC, Peterson BL, Morrison VA et al. Randomized phase 2 study of fludarabine with concurrent versus sequential treatment with rituximab in symptomatic, untreated patients with B-cell chronic lymphocytic leukemia: results from Cancer and Leukemia Group B 9712 (CALGB 9712). Blood 2003;101:6-14.

32. Byrd JC, Rai K, Peterson BL et al. Addition of rituximab to fludarabine may prolong progression-free survival and overall survival in patients with previously untreated chronic lymphocytic leukemia: an updated retrospective comparative analysis of CALGB 9712 and CALGB 9011. Blood 2005; 105:49-53.

33. Keating MJ, O'Brien S, Albitar M et al. Early results of a chemoimmunotherapy regimen of fludarabine, cyclophosphamide, and rituximab as initial therapy for chronic lymphocytic leukemia. J Clin Oncol 2005; 23:4.079-4.088.

Avaliação: Carlos Sergio Chiattone (publicado após acordo do Editor)

Conflito de interesse: Artigo derivado do II Encontro Brasileiro de Consenso da LLC.

Recebido: 23/01/2006

Aceito: 23/01/2006 